\title{
Evidence for multiple impurity bands in sodium-doped silicon MOSFETs
}

\author{
T. Ferrus 4 R. George, C. H. W. Barnes, N. Lumpkin, D. J. Paul, and M. Pepper \\ Cavendish Laboratory, University of Cambridge, Madingley Road, CB3 OHE, Cambridge, United Kingdom
}

(Dated: November 9, 2018)

\begin{abstract}
We report measurements of the temperature dependent conductivity in a silicon MOSFET that contains sodium impurities in the oxide layer. We explain the variation of conductivity in terms of Coulomb interactions that are partially screened by the proximity of the metal gate. The study of the conductivity exponential prefactor and the localisation length as a function of gate voltage have allowed us to determine the electronic density of states and has provided arguments for the presence of two distinct bands and a soft gap at low temperature.
\end{abstract}

PACS numbers: 71.23.Cq, 71.55.Gs, 71.55.Jv, 72.15.Rn, 72.20.Ee, 72.80.Ng, 73.20.At, 73.40.Qv

Since the invention of the silicon MOSFET, understanding the influence of impurities, especially sodium contamination, on device performance has been a priority and continues to provide a rich system for investigation by experimental and theoretical physicists alike. The electronic properties of sodium doped MOSFETs were first studied by Fowler and Hartstein ${ }^{1.2}$ in the 1970s. They reported a single, broad peak in the subthreshold drain current against gate voltage and attributed it to the formation of an impurity band induced by the presence of sodium ions near the $\mathrm{Si}_{-} \mathrm{SiO}_{2}$ interface. Further studies of narrow channel devices $(\sim 100 \mathrm{~nm})$ demonstrated a series of reproducible sharp peaks ${ }^{3}$, while later experiments found evidence for resonant tunneling between localised states in the channel $\stackrel{4.5 .6}{\text { For sufficiently low }}$ impurity concentrations, the overlap between neighbouring localized electron wavefunctions and consequently the hybridisation of their excited states is predicted to be reduced ${ }^{\mathbf{z}}$, splitting the single impurity band observed at high concentrations into the ground and excited bands as modeled by Ghazali $\stackrel{8}{\underline{ }}$ Increasing the resistivity of the silicon substrate reduces the scattering from acceptors at the $\mathrm{Si}-\mathrm{SiO}_{2}$ interface, allowing the possibility for such a band splitting to be experimentally observed in the transport. In this paper, we will present evidence for the observation of two separate impurity bands with a soft gap, based on analysis of the temperature dependent conductivity below $20 \mathrm{~K}$.

The device we used is a MOSFET fabricated on a (100) oriented p-silicon wafer and was subsequently patterned in the circular Corbino geometry to eliminate Hall voltages and possible leakage paths. The effective gate channel length and interior width were respectively $1 \mu \mathrm{m}$ and $346 \mu \mathrm{m}$. A high resistivity wafer $\left(10^{4} \Omega . \mathrm{cm}\right)$ provided a background concentration of less than $10^{12} \mathrm{~cm}^{-3}$ of boron corresponding to a mean distance between impurities of $1 \mu \mathrm{m}$. A $35 \mathrm{~nm}$ gate oxide was grown at $950^{\circ} \mathrm{C}$ in a dry, chlorine-free oxygen atmosphere. The phosphorous implanted and aluminium sputtered contacts were highly metallic and Ohmic at all temperatures investigated. Sodium ions were introduced onto the oxide surface by immersing the device in a $10^{-7} \mathrm{~N}$ solution of high purity sodium chloride (99.999\%) in de-ionised water. The surface of the chip was dried with nitrogen gas and an aluminium gate subsequently evaporated. To observe or remove the low temperature conductivity structures, the mobile ions are drifted through the oxide to the $\mathrm{Si}$ $\mathrm{SiO}_{2}$ interface, or returned to the $\mathrm{Al}-\mathrm{SiO}_{2}$ interface by applying either $\mathrm{a}+4 \mathrm{~V}$ or a $-4 \mathrm{~V}$ DC gate-substrate bias for $10 \mathrm{~min}$ at $65^{\circ} \mathrm{C}$ before the device is cooled down to helium temperature at which sodium looses its diffusivity in the oxide. All measurements were performed using standard low-noise lockin techniques with an amplifier of $10^{8} \mathrm{~V} / \mathrm{A}$. The AC excitation was maintained at $15 \mu \mathrm{V}$ with a frequency of $11 \mathrm{~Hz}$. Suitable RC filters were employed to eliminate any DC offset from the amplifier. The gate voltage was controlled by a high resolution digital to analog converter. All experiments were performed in an ${ }^{3} \mathrm{He}$ cryostat and the temperature was measured by a calibrated germanium thermometer.

Fig. 1 shows the conductivity $\sigma$ of our device at $300 \mathrm{mK}$ versus gate voltage $V_{\mathrm{g}}$ for the case where the sodium ions had been drifted to the $\mathrm{Si}_{-} \mathrm{SiO}_{2}$ interface. Two groups of peaks appear clustered around $V_{g}=-2 \mathrm{~V}$ and $-0.5 \mathrm{~V}$ and separated by a region of low conductivity and limited by noise. The origin of the peaks themselves will be discussed later. Following a $-4 \mathrm{~V}$ drift, no structure was detectable over the full range of gate voltages investigated but a difference in threshold voltage of $0.2 \mathrm{~V}$ was found at $77 \mathrm{~K}$ between the characteristics of the device following $+4 \mathrm{~V}$ and $-4 \mathrm{~V}$ drifts. This was attributed to the presence of mobile charges close to the $\mathrm{Si}_{-} \mathrm{SiO}_{2}$ interface at a concentration of $3.7 \times 10^{11} \mathrm{~cm}^{-2}$ corresponding to a mean impurity separation of $16 \pm 1 \mathrm{~nm}$. In a reference device where no sodium was introduced, no sub-threshold conductivity peaks and no shift of the threshold voltage appeared for any drift conditions investigated. Tunneling through the oxide and other leakage currents were discounted as the gate leakage current was below $50 \mathrm{fA}$ at $4.2 \mathrm{~K}$ and was approximately constant over the range of gate voltages used. We remark also that no hysteresis and thus no charging effects that have been reported in similar devices 9 were observed here.

The presence of two distinct ranges of $V_{\mathrm{g}}$ where peaks appear suggests the possibility of a split impurity band (Fig. 1). Such a splitting into a ground and an excited state is expected to happen for low-doping concentration $^{8.10}$ if one takes into account the overlaps 


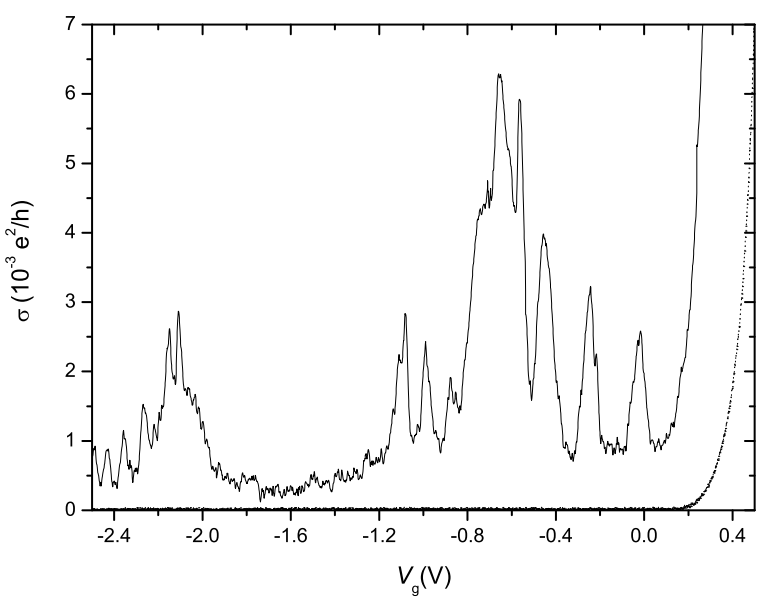

FIG. 1: The source drain conductivity versus gate voltage at $300 \mathrm{mK}$ following a $+4 \mathrm{~V}$ drift and a $-4 \mathrm{~V}$ drift (dotted line).

between impurity wavefunctions and uses a multi-band formalism 11 . The conductivity of a Si-MOSFET is not directly related to the density of states and the fact that we see two regions of high conductivity separated by a region of low conductivity is only an indirect indication that the device density of states consists of two bands separated by a gap. In fact, through the Kubo formalism, conductivity tends to be related to local paths through a disordered device but density of states is a global property. In order to show that the density of states splits into two bands, we have looked at the temperature dependence of the conductivity at a series of different gate voltages. For all gate voltages studied, the conductivity decreases non-monotonically as temperature is lowered (Fig. 2). In the range $1 \mathrm{~K}$ to $20 \mathrm{~K}$, we observe the characteristics of hopping conduction so that the conductivity $\sigma(T)$ is fitted to the generalised equation :

$$
\sigma=\sigma_{0} T^{-\gamma s} \mathrm{e}^{-\left(\frac{T_{0}}{T}\right)^{s}}
$$

where $T_{0}$ and $\sigma_{0}$ depend on gate voltage.

The best values for $\gamma$ and $s$ are found by minimizing the value of the reduced chi-square deviation $\chi^{2}$ with standard procedures. Figs 2(a)-(b) show the optimum values determined for four gate voltages, one point in the threshold region $\left(V_{g}=0.1 \mathrm{~V}\right)$, the upper band $\left(V_{g}=\right.$ $-0.24 \mathrm{~V})$, the gap $\left(V_{g}=-1.48 \mathrm{~V}\right)$ and the lower band $\left(V_{g}=-2.26 \mathrm{~V}\right)$. The resulting fits for $\sigma(T)$ are valid over three orders of magnitude in $\sigma$ (Fig. 3). Studies in gate voltage show that $\gamma=1.98 \pm 0.04$ and $s=0.39 \pm 0.02$ for $\mathrm{V}_{\mathrm{g}}$ below $0.25 \mathrm{~V}$. Above this point, the value of $s$ decreases rapidly towards $s=1 / 3$ for $T \geq 4 \mathrm{~K}$ and the range is better described by Mott hopping conduction ${ }^{12}$. It is worth noticing that the smallest values of $s$ are obtained for band centre regions $-0.8 \mathrm{~V}<V_{\mathrm{g}}<-0.5 \mathrm{~V}$ and $-2.2 \mathrm{~V}<V_{\mathrm{g}}<-2.1 \mathrm{~V}$ for which the hopping lengths are relatively smaller and the Coulomb interactions weaker.

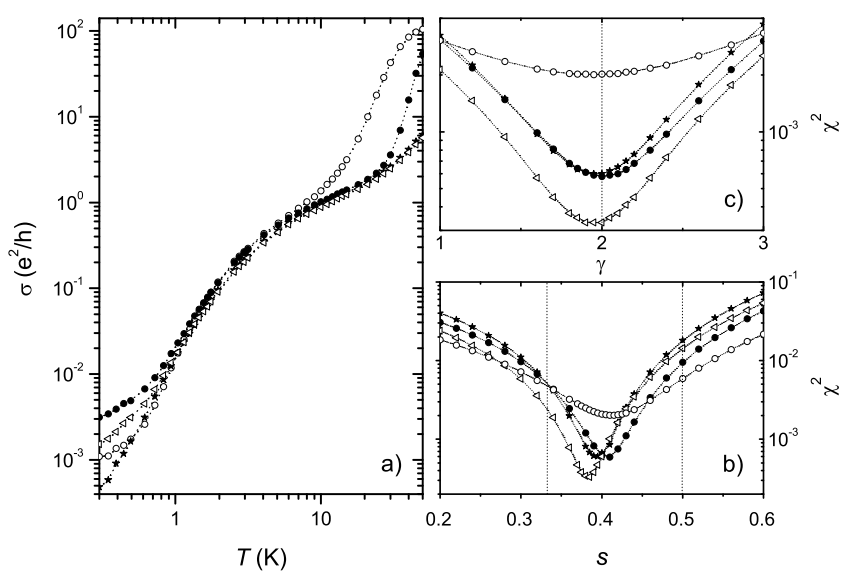

FIG. 2: a) Temperature dependence of the conductivity for $V_{\mathrm{g}}=0.1(\circ),-0.24(\bullet),-1.48 \mathrm{~V}(\star)$ and $\left.-2.26(\triangleleft), \mathrm{b}\right)$ Variation of the reduced $\chi^{2}$ with $s$ for $\gamma=2$ for the gate voltages listed showing minima at $s=0.412,0.406,0.394$ and 0.385 respectively and c) Variation of the reduced $\chi^{2}$ with $\gamma$ for $s$ equal to the optimum value found in 2(b), for the appropriate gate voltages, displaying consistency with a minimum at $\gamma=2$ (dotted line). Lines in b) represent the exponent for the Mott hopping regime (left) and Efros and Schklovskii regime (right).

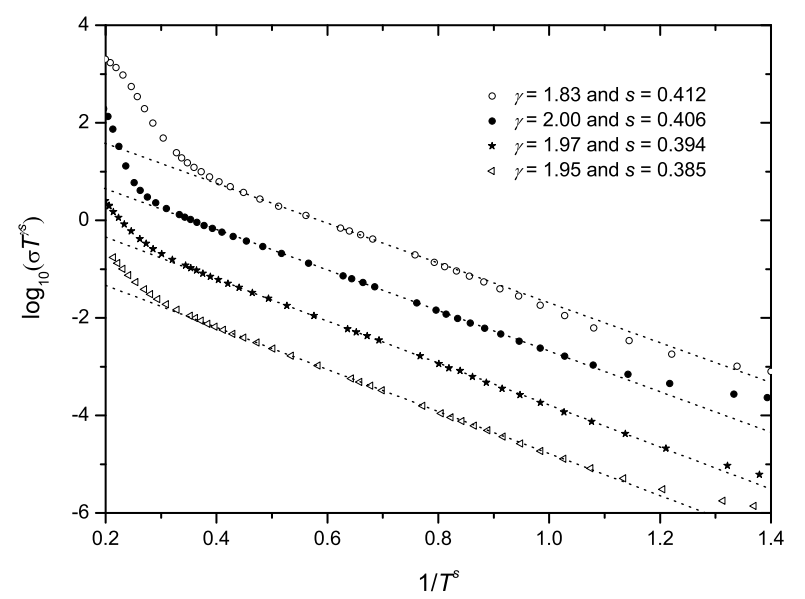

FIG. 3: Temperature dependence of the conductivity for $V_{\mathrm{g}}=0.1(\circ),-0.24(\bullet),-1.48 \mathrm{~V}(\star)$ and $-2.26(\triangleleft)$ for the optimum values of $\gamma$ and $s$ defined in Eq. 1. For clarity, curves are shifted downward respectively by $0,1,2$ and 3 from their original values.

We emphasise that the use of temperature dependent exponential prefactors allows the fine distinction between Mott $(s=1 / 3)$, Efros-Schklovskii $(s=1 / 2)^{13}$ and the regime under study $(s \sim 0.39)$. The finding that $\gamma \sim 2$ is consistent with the formulation for $\sigma(T)$ given by Allen and Adkins 14.15 if it is rederived for the $2 \mathrm{D}$ case. This gives the conductivity in terms of both the localization length $\xi$ and the density of states at the Fermi level $n\left(E_{F}\right)$. Accounting for the fact that Coulomb interactions between electrons in different localized states mod- 
ifies the density of states close to the Fermi level so that $n(E)=N_{0}\left|E-E_{F}\right|^{p}$, we obtain :

$$
\begin{gathered}
\sigma=\sigma_{0} T^{-\gamma\left(\frac{p+1}{p+3}\right)} \mathrm{e}^{-\left(\frac{T_{0}}{T}\right)^{\frac{p+1}{p+3}}} \text { with } \gamma=2 \\
\sigma_{0}=\frac{A_{0}}{\xi^{2}(p+3)^{2}} T_{0}^{2\left(\frac{p+1}{p+3}\right)} \\
k_{B} T_{0}=\left(\frac{p+3}{p+1}\right)^{\frac{p+3}{p+1}}\left[\frac{(p+1)^{3}}{\pi N_{0} \xi^{2}}\right]^{\frac{1}{p+1}}
\end{gathered}
$$

where $A_{0}$ is a constant depending on the electronic properties of bulk silicon and $k_{B}$ the Boltzmann constant.

The conditions given by Allen ${ }^{14}$ for the use of the equations Eqs. 2-4 are satisfied in our device. Recent calculations showed that sodium ions in the oxide may trap either one or two valence electrons against the Si$\mathrm{SiO}_{2}$ interface and that the wavefunctions of the localized states remain hydrogen-like ${ }^{16}$ Also, hopping conduction is present in our device but Coulomb interactions are such as the only mobile electrons are found in an energy band of few $k_{B} T$ around the Fermi level. The resulting Coulomb gap for $s=0.39$ (i.e. $n(E) \sim\left|E-E_{F}\right|^{0.30}$ ) is much sharper than for the Efros regime where $n(E) \sim$ $\left|E-E_{F}\right|$. This behaviour has been predicted by Blanter and Raikh ${ }^{17}$ while studying $2 \mathrm{D}$ systems localized by disorder. They have shown that a metallic gate close to the interface provides image potentials that modify the density of states to the form $n(E)=n\left(E_{F}\right)+N_{0}\left|E-E_{F}\right|^{1 / 3}$ at $T=0 \mathrm{~K}$ and thus gives an exponential dependence of $T^{-0.4}$ for the conductivity. This behaviour is explained by the fact that the oxide thickness plays the role of the screening length and that initial and final states become electrostatically independent at low temperature when the hopping length $R$ becomes greater than twice the oxide thickness $d$. This then produces a crossover from the Efros to the Mott regime. The localization length in our device was approximately estimated by fitting the conductivity with $p=0$, using the Mott formula for $k_{B} T_{0}$ and taking the $2 \mathrm{D}$ value for the density of states. For $V_{g}=-0.4 \mathrm{~V}$, this gives $\xi \sim 22 \mathrm{~nm}$ and a hopping length $R \sim 63 \mathrm{~nm}$ at $1 \mathrm{~K}$. Our device has a gate oxide of $d=35 \mathrm{~nm}$ and the Coulomb interactions may be screened by the electrostatic gate as $R \sim 2 d$. This demonstrates the device may well be in the regime described by Blanter and Raikh. Fits of the conductivity using $\gamma=2$ and $s=0.4$ are still valid for $V_{g}$ smaller than $0.25 \mathrm{~V}$ and from $1 \mathrm{~K}$ to $18 \mathrm{~K}$ typically, so we will proceed with these values. In this regime, the density of states at $E_{F}$ is given by :

$$
n\left(E_{F}\right)=n_{0}\left(E_{F}\right)-\frac{2 \pi n_{0}\left(E_{F}\right)^{2} d e^{2}}{4 \pi \epsilon_{0} \epsilon_{r}} \nu
$$

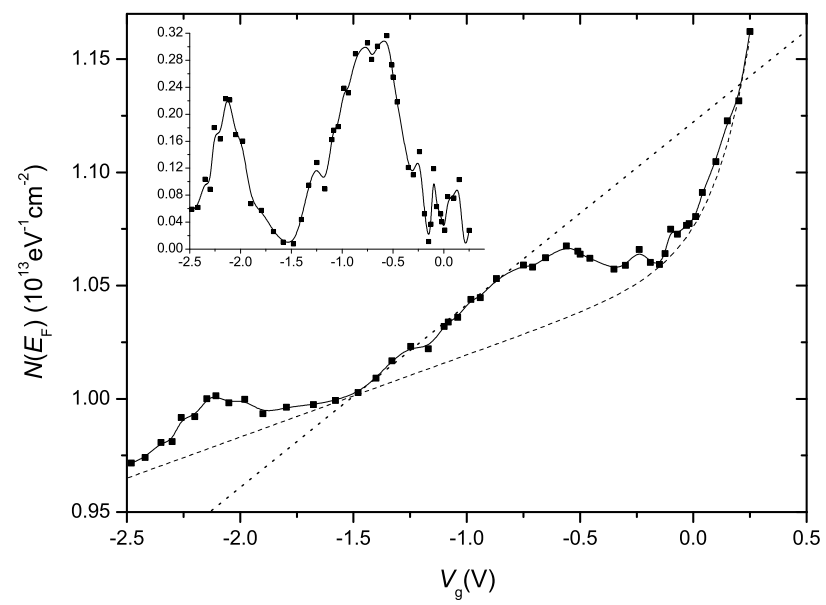

FIG. 4: Variation of the DOS at $E_{F}$ with gate voltage. The dashed and dotted lines represent respectively the background density due to the conduction band tail and the upper band tail. The inset is the relative variation of the DOS to the background in the same units.

$$
n_{0}\left(E_{F}\right)=\left(\frac{N_{0}}{2 \pi}\right)^{\frac{1}{2}}\left(\frac{4 \pi \epsilon_{0} \epsilon_{r}}{2 e^{2} d^{2}}\right)^{\frac{1}{3}}
$$

where $\epsilon_{r}$ is the permittivity of silicon.

These equations have been derived at $T=0 \mathrm{~K}$. At higher temperature but for $k_{B} T \ll V(d)$, where $V$ is the coulomb potential energy, the gap is partly filled. The Coulomb interactions then become negligible for energies below $V(d)$ and the density of states saturates such as $n_{0}\left(E_{F}\right)=n_{0}\left(E_{F}+V(d)\right), 18$ This condition decreases the value of $\nu$ from 1 down to 0.05 . Combining (3) and (4), the parameters $N_{0}$ and $\xi$ were expressed in terms of $T_{0}$ and $\sigma_{0}$, values easily accessed experimentally. The density of states was extracted using equation (5) and (6). To find the value of $A_{0}$, we used $\Xi=8.91 \mathrm{eV}$ for the effective deformation potential of acoustic phonons ${ }^{19}$ as well as $3800 \mathrm{~m} . \mathrm{s}^{-1}$ for the speed of surface acoustic phonons in silicon ${ }^{20}$. This gives $A_{0}=6.69 \times 10^{-19} e^{2} \cdot h^{-1} \cdot \mathrm{m}^{2}$.

The density of states is shown in Fig. 4 . The value we find is more than one order of magnitude lower than the pure $2 \mathrm{D}$ case $\left(\sim 1.6 \times 10^{14} \mathrm{eV}^{-1} . \mathrm{cm}^{-2}\right.$ with valley degeneracies). The large background is predominantly due to the conduction band tail which spreads over the full range of $V_{g}$ studied. The upper band also has a significant tail. The presence of density of states tails is a common occurance in disordered systems with low impurity concentration and localized wavefunctions 21.22 .23 but its linear shape for $V_{g}<0 \mathrm{~V}$ is unusual. It has been attributed to the formation of regions of constant local potential energy at the $\mathrm{Si}_{-} \mathrm{SiO}_{2}$ interface and containing a random number of charges ${ }^{24}$ Two regions of higher density are superimposed on the background density and correspond to the upper and lower groups of peaks. This confirms that the structure observed in the conductivity is due to the presence of two separate bands. By numerically subtracting the background density and 


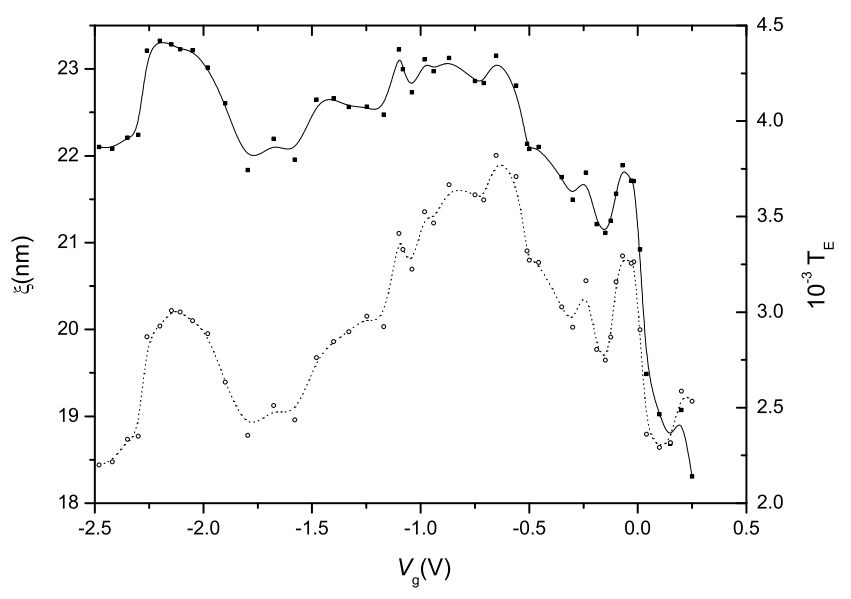

FIG. 5: Variation of the localisation length $(\bullet)$ and the transmission coefficient at $1 \mathrm{~K}(\mathrm{\circ})$ with gate voltage.

integrating over the appropriate gate voltage and by supposing a linear relation between the gate voltage and the surface potential energy, we estimate the upper band contains approximately 3 times the number of states as the lower band. This value is an upper bound as energetically deeper states could not be accessed experimentally.

The variation of the localization length $\xi$ (Fig. 5) follows that of the density of states, showing the two bands do correspond to the more conductive regions. The value of $\xi$ decreases rapidly when approaching the threshold voltage. This is expected as the region between $V_{g}=0 \mathrm{~V}$ and $V_{g}=0.4 \mathrm{~V}$ is both in the band tails of the conduction band and the upper band, the conduction band edge being well above $0.4 \mathrm{~V}$. Thus, this region is still a region of strong localization. Nevertheless, the value of $\xi$ is expected to rise once the Fermi energy crosses the conduction band edge. Finally, taking into account fitting errors as well as the discrepancy in the value of the deformation potential, we estimate the localization length within $5 \%$ and the density of states within $6 \%$. From the same derivation which gave (1), (2) and (3), the transmission coefficient is obtained :

$$
\ln \left(T_{E}\right)=-\frac{2}{p+3}\left(\frac{T_{0}}{T}\right)^{\frac{p+1}{p+3}}
$$

The experimental variation of the transmission coefficient in gate voltage (Fig. 5) shows that the conductivity of the two bands mostly comes from the higher mobility of the states of energies within the upper and lower bands and less from the increase in the density of states.

In conclusion, we have observed an unusual hopping regime with an exponent 0.4 which results from the screening of the Coulomb interactions by the metal gate. We have shown that, consequently, both the localization length and the density of states can be extracted from the temperature dependence of the conductivity. This analysis has given strong evidence for the existence of two separate bands and a soft gap at low temperature. This may result from the splitting of the impurity band into a lower and an upper band in presence of Coulomb interactions. The formation of the two bands results itself from the presence of a low concentration of sodium impurities close to the $\mathrm{Si}_{-} \mathrm{SiO}_{2}$ interface. The conditions for the observation of such a formation may be the creation of deep but well separated impurity potentials at the interface, resulting in electron localization. Finally, the electron screening may be sufficiently weak and the disorder not too strong to allow a Mott-Hubbard transition to take place. The two bands could then possibly be Hubbard bands.

We would like to thank Drs T. Bouchet and F. Torregrossa from Ion Beam System-France for the process in the device as well as funding from the U.S. ARDA through U.S. ARO grant number DAAD19-01-1-0552.
* Electronic address: taf25@cam.ac.uk

1 F. F. Fang, A. B. Fowler, Phys. Rev. 169, 619 (1967)

2 A. Hartstein, A. B. Fowler, Phys. Rev. Lett. 34, 1435 (1975)

3 A. B. Fowler, A. Hartstein and R. A. Webb, Phys. Rev. Lett. 48, 196 (1982)

4 A. B. Fowler, G. L. Timp, J. J. Wainer and R. A. Webb, Phys. Rev. Lett. 57, 138 (1986)

5 T. E. Kopley, P. L. McEuen and R. G. Wheeler, Phys. Rev. Lett. 61, 1654 (1988)

6 D. Popovic, A. B. Fowler and S. Washburn, Phys. Rev. Lett. 67, 2870 (1989)

7 C. Erginsoy, Phys. Rev. 80, 1104 (1950)

8 A. Ghazali, A. Gold and J. Serre, Phys. Rev. B 39, 3400 (1989)

9 V. Ioannou-sougleridis, A. G. Nassiopoulou and A. Travlos, Nanotechnology, 14, 11174 (2003)

10 J. Serre and A. Ghazali and A. Gold, Phys. Rev. B 39, 8499 (1989)
11 J. R. Klauder, Ann. Phys. (N.Y.) 14, 43 (1961)

12 N. H. Mott, J. Non-Cryst. Solids 1, 1 (1969)

13 A. L. Efros and B. I. Schklovskii, J. Phys. C 8, L49 (1975)

14 F. R. Allen and C. J. Adkins, Phil. Mag. 26, 1027 (1972)

15 R. Mansfield, S. Abboudy and P. Fozooni, Phil. Mag. B 57, 6, 777 (1988)

16 C. H. W. Barnes and A.V. Moroz, to be published

17 Y. M. Blanter and M. E. Raikh, Phys. Rev. B 63, 075304 (2001)

18 I. L. Aleiner and B. I. Shklovskii , Phys. Rev. B 49, 13721(1994)

19 M. V. Fischetti and S. E. Vaux, J. Appl. Phys. 80, 2234 (1996)

20 G. T. Andrews, M. J. Clouter and J. Zuk, Semicond. Sci. Technol. 19, 1306 (2004)

21 B. I. Halperin and M. Lax, Phys. Rev. 148, 722 (1966)

22 J. Zittartz and J. S. Langer, Phys. Rev. 148, 741 (1966)

23 E. O. Kane, Phys. Rev. 131, 79 (1963)

24 E. Arnold, Appl. Phys. Lett., 25, 705 (1974) 